\title{
Production of biofuels and biochemicals: in need of an ORACLE
}

\section{Ljubisa Miskovic and Vassily Hatzimanikatis}

Laboratory of Computational Systems Biotechnology, Ecole Polytechnique Federale de Lausane (EPFL), CH 1015 Lausanne, Switzerland

The engineering of cells for the production of fuels and chemicals involves simultaneous optimization of multiple objectives, such as specific productivity, extended substrate range and improved tolerance - all under a great degree of uncertainty. The achievement of these objectives under physiological and process constraints will be impossible without the use of mathematical modeling. However, the limited information and the uncertainty in the available information require new methods for modeling and simulation that will characterize the uncertainty and will quantify, in a statistical sense, the expectations of success of alternative metabolic engineering strategies. We discuss these considerations toward developing a framework for the Optimization and Risk Analysis of Complex Living Entities (ORACLE) - a computational method that integrates available information into a mathematical structure to calculate control coefficients.

\section{Biofuels and biochemicals: a multi-objective optimization problem}

Nearly 20 years ago, Tong and Cameron classified the applications of metabolic engineering into five main areas: (i) improved production and utilization of chemicals already produced/used by the host; (ii) extended substrate range for growth and production; (iii) addition of new catabolic activities for the degradation of toxic chemicals; (iv) production of chemicals new to the host; and (v) modification of the cell [1]. In the development of microorganisms for fuels and chemicals, one must consider and optimize almost all of these objectives simultaneously. For example, the economics of the fuels and commodity chemicals will require a host that is able to overproduce a fuel or chemical (native or new to the organism) from a broad range of carbon substrates, some of them not used previously by this organism, with high specific rates and with near-theoretical yields [2]. All of these issues become more challenging when we consider the use of new hosts for engineering [3,4], where there is not enough genomic information, the genetic tools for metabolic engineering are limited, and extensive knowledge about their physiology is lacking.

The development of microorganisms for fuels and chemicals will also require the fine-tuning of carbon flows and redox balance. All of the biofuels currently considered require delicate manipulation of the carbon flows in the

Corresponding author: Hatzimanikatis, V. (vassily.hatzimanikatis@epfl.ch). central metabolism, and any intervention in these pathways can have significant implications for the cellular physiology. Redox balance, energy charge and cofactor levels are also important because they are involved in many of the pathways for the production of biofuels and acids. For example, these pathways involve reactions that produce and consume redox potential, and their directionality is determined by the $\mathrm{NAD}^{+} / \mathrm{NADH}$ ratio. Metabolic engineering of these pathways could alter the balance of redox and coenzyme cofactors, thereby reducing the productivity and the yield, leading to the production of byproducts that could increase the cost of downstream processing [2,5-7].

\section{Guidance for metabolic engineering}

Metabolic engineering design requires identification of rate-limiting steps in metabolic pathways. A large amount of effort has been invested in the quantification of the metabolic fluxes within cells, using methods such as metabolic flux analysis [8-10]. However, one of the limitations of these methods is that they provide only a snapshot of the fluxes and do not quantify the responses of metabolic networks to the changes in the metabolic parameters or the process parameters, such as oxygen and carbon substrate limitation, or stress agents, such as organic acids and $\mathrm{pH}$. Similar to flux analysis, metabolite profiling, although provides information about the redox state of the cells, it is only a quantitative snapshot of the metabolite levels and, currently, only represents a partial set of the metabolites in pathways of interest [11-14].

Mathematical modeling and computational analyses have been used successfully for metabolic engineering [15-20]. Constrained-based flux balance analysis has been one of the most widely used approaches. Sophisticated computational methods, such as OptKnock and OptStrain, have been developed for the analysis of constrained-based genome-scale models and they been very successful in identifying targets for metabolic engineering $[16,17,21,22]$. However, methods and approaches that use constrained-based flux balance analysis can be used only for the optimization through gene addition and deletion.

Identification of the rate limiting steps requires either a series of a large number of experiments or a mathematical description of the metabolic networks that takes kinetic properties into account [23]. One of the unparalleled advantages in the use of mathematical models that account for enzyme kinetics is that they allow us to estimate how we can manipulate the metabolite levels and, 


\section{Box 1. Elements of MCA}

Within MCA, two important quantities are defined: flux control coefficients (FCC) and concentration control coefficients (CCC). In turn, these quantities are determined by the elasticities, which quantify the strength of interactions of the enzymes with their corresponding substrates, products, inhibitors and activators. Control coefficients capture the sensitivity of the metabolic networks to metabolic parameters, and although they are based on mathematical theories of local sensitivity (i.e. they account for small changes in parameters and responses around a reference state), they are useful for understanding the properties of the metabolic pathways toward metabolic engineering. The prediction of cellular responses to more realistic ranges of manipulation of metabolic and process parameters can be improved through the application of methods that extend MCA to large changes in parameters and responses [31].

The control coefficients are defined as:

- Flux control coefficient (FCC)

$$
C_{p}^{v}=\frac{d \ln v}{d \ln p}=\frac{\frac{d v}{v}}{\frac{d p}{p}} \approx \frac{\frac{\Delta v}{v}}{\frac{\Delta p}{p}}
$$

- Concentration control coefficient (CCC)

$$
C_{p}^{x}=\frac{d \ln x}{d \ln p}=\frac{\frac{d x}{x}}{\frac{d p}{p}} \approx \frac{\frac{\Delta x}{x}}{\frac{\Delta p}{p}}
$$

Sensitivity of reactions to the variation of metabolite concentration or a parameter is quantified by:

- Metabolite elasticity

$$
\varepsilon_{x}^{v}=\frac{\partial \ln v}{\partial \ln x}=\frac{\frac{\partial v}{v}}{\frac{\partial x}{x}}
$$

- Parameter elasticity

$$
\pi_{p}^{v}=\frac{\partial \ln v}{\partial \ln p}=\frac{\frac{\partial v}{v}}{\frac{\partial p}{p}}
$$

consequently, the redox and energetic states of the cell (i.e. energy charge) using metabolic engineering.

Metabolic control analysis (MCA) has been proposed as a methodology for quantifying the response of metabolic systems to changes in cellular and process parameters, such as enzyme activities, $\mathrm{pH}$ and oxygen availability (Box 1) [24-30]. However, for MCA to be a predictive, guiding methodology, it requires a kinetic model; otherwise, the number of experiments required to identify the rate-limiting steps might be even larger than the number of the experiments required for solving the problem at hand.

\section{Uncertainty in biological systems}

A predominant issue in the development of kinetic models of metabolic networks is the lack of available information
Metabolite elasticities quantify also the strength of interaction between metabolites and enzymes. For a simple three-step reaction:

$\mathrm{E}+\mathrm{S} \underset{k_{1 b}}{\stackrel{k_{1} f}{\rightleftarrows}} \mathrm{ES} \underset{k_{2 b}}{\stackrel{k_{2} f}{\rightleftarrows}} \mathrm{EP} \underset{k_{3 b}}{\stackrel{k_{3} f}{\rightleftarrows}} \mathrm{E}+\mathrm{P}$

the common rate equation is:

$=\frac{V_{m}^{f} \bar{S}-V_{m}^{b} \bar{P}}{1+\bar{S}+\bar{P}}$

with: $V_{m}^{f}=\frac{k_{2 f} k_{3 f} E_{T}}{k_{2 f}+k_{3 f}+k_{3 b}}, V_{m}^{b}=\frac{k_{1 b} k_{2 b} E_{T}}{k_{2 f}+k_{1 b}+k_{2 b}}, \bar{S}=\frac{S}{K_{m 1}}$, and $\bar{P}=\frac{P}{K_{m 1}}$,

where: $K_{m 1}=\frac{k_{2 f} k_{3 f}+k_{1 b} k_{3 f}+k_{1 b} k_{2 b}}{k_{1 f}\left(k_{2 f}+k_{3 f}+k_{2 b}\right)}, K_{m 2}=\frac{k_{2 f} k_{3 f}+k_{1 b} k_{3 f}+k_{1 b} k_{2 b}}{k_{3 b}\left(k_{2 f}+k_{1 b}+k_{2 b}\right)}$,

and $E_{\mathrm{T}}=E+E S+E P$.

From the rate expression, the elasticities are expressed as:

$\varepsilon_{\bar{S}}^{v}=\frac{v_{f}}{v} \frac{1+\bar{P}}{1+\bar{S}+\bar{P}}+\frac{v_{b}}{v} \frac{\bar{S}}{1+\bar{S}+\bar{P}}$

$\varepsilon_{\bar{P}}^{v}=-\frac{v_{f}}{v} \frac{1+\bar{P}}{1+\bar{S}+\bar{P}}-\frac{v_{b}}{v} \frac{\bar{S}}{1+\bar{S}+\bar{P}}$

These elasticities can also be expressed as functions of the enzyme states and the displacements from the equilibrium of individual reaction steps $\left\{\gamma_{1}, \gamma_{2}, \gamma_{3}\right\}$ :

$\varepsilon_{S}^{v}=\frac{\frac{E}{E_{T}}+\gamma_{2} \gamma_{3} \frac{E S}{E_{T}}+\gamma_{3} \frac{E P}{E_{T}}}{1-\gamma_{1} \gamma_{2} \gamma_{3}}$

and

$\varepsilon_{P}^{v}=\frac{\gamma_{1} \gamma_{2} \gamma_{3} \frac{E}{E_{T}}+\gamma_{2} \gamma_{3} \frac{E S}{E_{T}}+\gamma_{3} \frac{E P}{E_{T}}}{1-\gamma_{1} \gamma_{2} \gamma_{3}}$

The displacements from the equilibrium of individual reaction steps relate to the displacement of the overall reaction, $\Gamma$, through the product $\Gamma=\gamma_{1} \gamma_{2} \gamma_{3}$.

and the uncertainty associated with such information, such as metabolic fluxes and kinetic properties of enzymes. The uncertainty in the study of metabolic pathways can be classified in two types: structural and quantitative (Table 1). 'Structural uncertainty' deals with the lack of knowledge concerning the stoichiometry and the kinetic laws of the enzymes in the pathways. For most of the common organisms, such as Escherichia coli, Bacillus subtilis and Saccharomyces cerevisiae, the stoichiometry of the pathways is well-characterized; however, gaps exist in the pathways of many newly sequenced organisms (a list of more than 2000 completed or ongoing sequencing projects is available at: www.jgi.doe.gov/genome-projects/), and the kinetics of their enzymes are completely unknown [32-35]. Furthermore, the kinetic parameters of the

Table 1. Information source and uncertainty characterization

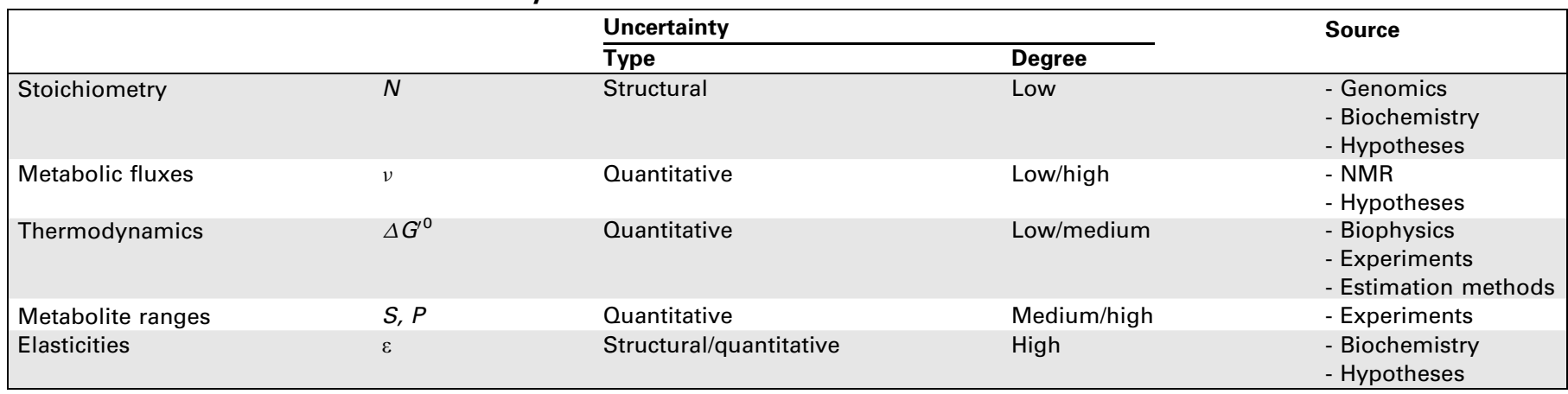


enzymes are usually known as 'apparent $K_{\mathrm{m}}$ values', but not as detailed kinetic mechanisms. In addition, enzyme kinetics are usually characterized in vitro, and it is possible that the kinetics will differ in a crowded intracellular (in vivo) environment [36,37].

'Quantitative uncertainty' is associated with the information about flux distributions, thermodynamic information, metabolite concentration and elasticities (Table 1). Despite advances in methods for the quantification of metabolic fluxes, there are still significant errors in their measurements. The thermodynamic properties of many reactions are also unknown and are based on group contribution methods, which themselves contain both experimental and estimation uncertainty [38]. Metabolite measurements carry the most uncertain information with respect to both their concentration levels and the number of metabolites we can measure.

Uncertainty is a common problem in the physical and chemical sciences and engineering [39]. Several methods and approaches exist within these fields that allow uncertainty modeling and quantification. Some of these methods have been used in the analysis of metabolic and signaling networks and, as a result, have provided some insight into the properties of the networks as well as guidance for metabolic engineering [40-47]. The challenges in the development of uncertainty analysis are in the modeling and simulation of uncertainty. With respect to kinetic models of chemical and biochemical systems, one must sample the kinetic parameters multiple times, calculate the properties of a population of the system, solve large systems of nonlinear equations, and perform a statistical analysis to characterize the properties of the system population [48-54]. This can be computationally infeasible because: (i) the ranges of the parameter values are not known or they are very large; (ii) the size and nonlinearities introduce computational difficulties; and (iii) reliable statistics will require a computationally prohibiting number of samples. Therefore, we need to develop methods for modeling and analysis of uncertainty in biological systems that will address these issues by integrating biological data from different levels and maximizing the extraction of information from these data. These modeling and analysis methods will provide guidance and alternatives for metabolic engineering, as discussed herein.

\section{Optimization and Risk Analysis of Complex Living Entities (ORACLE)}

Wang and Hatzimanikatis [40-42] have proposed an approach based on MCA frameworks that uses uncertainty analysis methods for the study of metabolic pathways and address most of the previously mentioned issues. The method, called ORACLE (optimization and risk analysis of complex living entities), is based on a sampling computational procedure and involves several steps where the available information is integrated into a mathematical structure and the control coefficients are calculated. Whenever this information is missing, a Monte Carlo sampling method is used. The main advantage of ORACLE stems from the finding that enzyme elasticities can be estimated if the distribution of the enzyme between the different mechanistic states is known. For example, in the simplest enzyme mechanism of a reversible unimolecular reaction, the elasticity depends on the displacement of the enzyme from equilibrium and on the distribution of the enzyme between three states: free enzyme, enzymesubstrate complex and enzyme-product complex. This observation led to the reconsideration of the uncertainties in the enzyme state space instead of the kinetic parameter space. This reformulation gives the advantage that one can derive the elasticity values by sampling the enzyme state space, which, unlike the parameter space, is very well bounded between 0 and 1 . In this way, the distributions of the elasticities for a given displacement from the thermodynamic equilibrium can be derived in an efficient manner.

The application of ORACLE and any similar methods must follow several steps that integrate biological information from different levels and sources; in doing so, these approaches guarantee that the analysis is consistent with biological and physical constraints, such as metabolite ranges and reactions thermodynamics. Within ORACLE the following procedure is considered:

Step 1. Definition of the stoichiometry, which can be obtained from biochemical data or genome reconstruction analysis [32-35]. It can also be hypothesized in the form of possible pathways when starting pathways are missing, or if a comparison between alternative chemical routes is desired.

Step 2. Estimation of the flux profiles using information from metabolomics analysis. Estimation can also be based on hypotheses about desirable flux distributions in an engineered pathway $[8,9,19]$.

Step 3. Estimation of the standard free energy of reactions. This is based on the available experimental information, or it can be estimated using group contribution methods $[38,55,56]$.

Step 4. Sampling of metabolite levels. For some metabolites in the system, measurements specific to the experimental system or estimates from experiments under similar physiological conditions might be available [11-14]; these estimates can provide the bounds for the computational sampling of the metabolites. In addition, the information from Step 2 will determine the direction that reversible reactions will operate and, combined with the information from Step 3, will be used for sampling the metabolite levels without violating thermodynamic and directionality constraints [57-59]. Step 5. Computation of the elasticities will be performed through the sampling of the enzyme states (Box 1). In general, elasticities depend on the displacement of the enzymes from the thermodynamic equilibrium. Therefore, for a given sample of displacement derived from Step 4, a distribution of elasticities through the sampling of the enzyme states is calculated.

Step 6. Consistency checks and pruning are tests that consider the stability of the system and the consistency with experimental information. For example, it might be known that the response of a metabolic flux to the changes in the activity of an enzyme is inconsistent with the control coefficients in some samples (Box 2). 


\section{Box 2. Information refinement}

As the available information increases, the distribution of the computed control coefficients can be refined (Figure I). If it is experimentally observed that the control coefficient of the flux $V_{x}, C_{p}^{V_{x}}$, is estimated to be larger than 0.3 , then the generated elasticities that lead to values of
$C_{p}^{V_{x}}$ that do not satisfy the constraint are rejected. The pruning of these elasticities at the same time refines the distributions of the other control coefficients as illustrated by a sharper mode of the distribution of the control coefficient of the flux $V_{y}, C_{p}^{V_{x}}$.

\section{Information refinement}
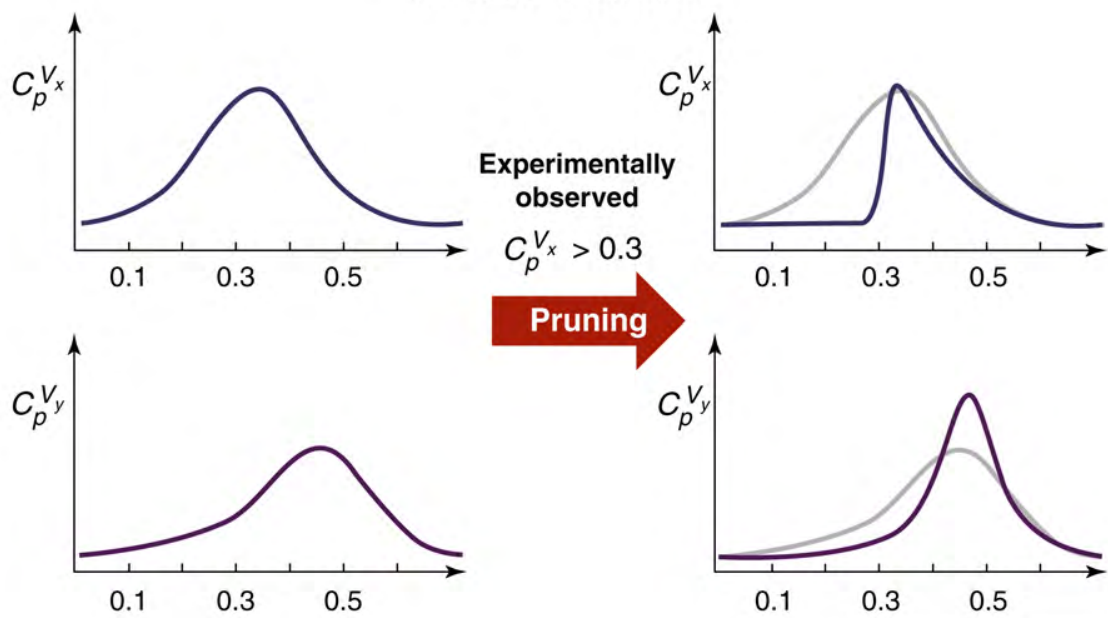

Pruning

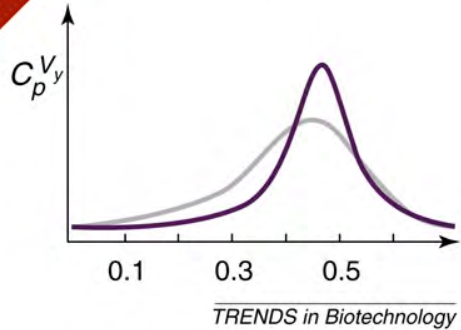

Figure I.

Step 7. Populations of control coefficients are calculated based on a well-established framework for MCA [41] and are then stored.

Step 8. Statistical analysis, data mining and visualization. The populations from Step 7 are analyzed using nonparametric statistics and data mining to rank the enzymes with respect to their impact on the objectives [60-62].

Although the proposed framework is designed around MCA, any alternative approach for metabolic engineering under uncertainty must consider the same steps and the associated issues: system modeling, uncertainty modeling, computational efficiency, statistical analysis and visualization.

\section{Prediction versus expectation}

The results from the application of the ORACLE or similar methods should not be viewed as predictions in the strict engineering sense; instead, they are predictions in a statistical manner, as expectations of success of the metabolic engineering targets they identify. Rather than providing a single solution, the analyses offer a set of alternative solutions that are evaluated with respect to their uncertainty, which itself is the propagation of the uncertainty in the available information. One of the benefits of this approach is that the alternative solutions can be given back to the experts who can evaluate them based on their 'expert opinion' - a type of information that cannot be modeled using conventional mathematical and statistical modeling methods.

The ability to screen for and formulate alternative strategies is crucial because the analysis generates a very large set of data with many variables and dimensions (multiple control coefficients). Similar to certain problems in data mining and analysis, the formulation of the proper question will dictate the methods and the tools used for such analysis. This situation is very similar to the problem facing biotechnology with 'omics' information. Here, the opportunity exists to adapt omics methodologies for analysis and visualization of information from the uncertainty analysis of the control distribution in metabolic networks. The metabolic control analysis of the central carbon pathways in yeast has been one of the first applications of this kind of methodology [41,42].

\section{A case study: ethanol production in yeast}

ORACLE has been used for the analysis of ethanol production by $S$. cerevisiae [41,42]. The only experimental

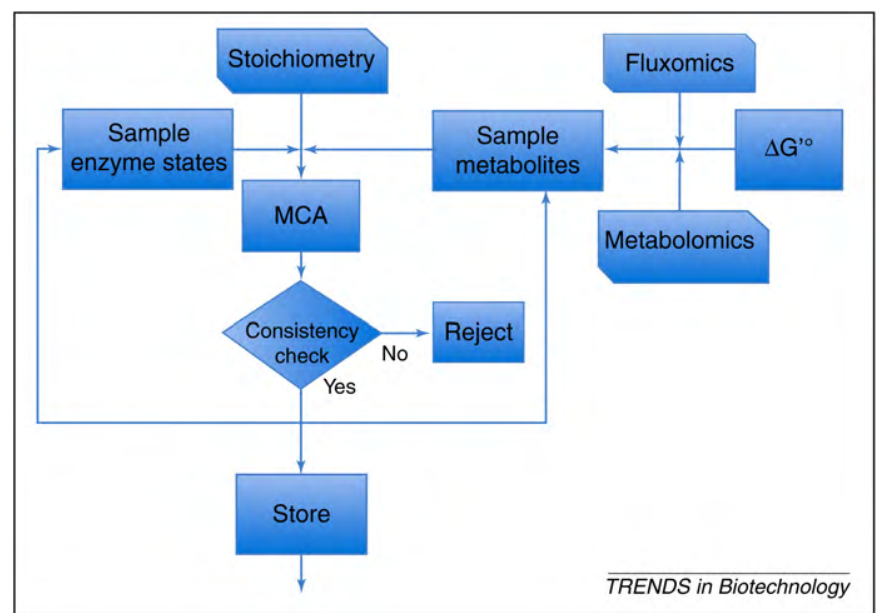

Figure 1. Computational procedure for uncertainty analysis of metabolic networks within ORACLE. Although the analysis revolves around MCA, the procedure described here should be the same for uncertainty analyses, which use alternative mathematical modeling frameworks. 


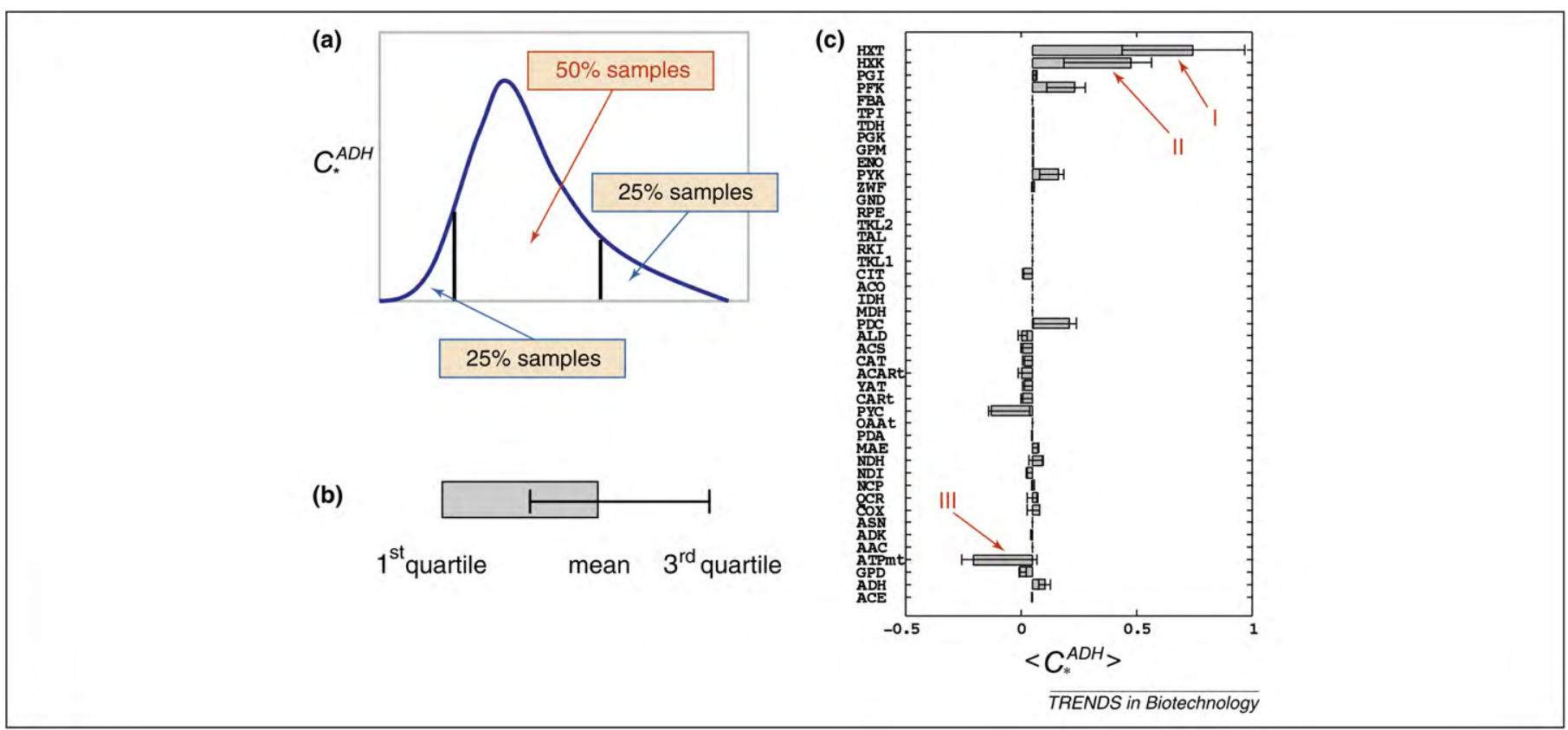

Figure 2. ORACLE analysis of the ethanol productivity in S. cerevisiae [42]. (a) The distribution of the flux control coefficients of ethanol production with respect to changes in the activities of the enzymes in the central carbon pathways, $C_{*}^{A D H}$, were computed and analyzed. (b) The mean value and the first and third quartiles were calculated; these values are plotted as box plots, and the bars that correspond to the quartiles provide visualization of the form (skewness) of the distribution of control coefficients. (c) A very small number of enzymes and cellular activities has a significant impact on the specific ethanol productivity. The three most statistically important enzymes and process are: (i) glucose transport (HXT); (ii) hexokinase (HXK); and (iii) ATP maintenance (ATPmt). Figure 2c adapted with permission from Ref. [42].

information used in this analysis was the pathway stoichiometry and the distribution of the metabolic fluxes [10]. Standard kinetic expressions (not kinetic parameters) were used for the enzymes, and metabolite concentrations were sampled within typical physiological ranges. In these studies, a mathematical model of the central carbon pathways in yeast was constructed, comprising 55 metabolites and 66 reactions, grouped into seven major pathways. It also included the compartmentalization of the reactions in the mitochondria and the transport reactions across the mitochondrial membrane. A reliable kinetic model for this system does not exist, and it is difficult to construct because the kinetic properties of such a large number of reactions are not known and the available experimental information does not allow reliable parameter estimation.

In this case study, the ORACLE methodology (Figure 1) was applied, using stoichiometry previously presented [10] for the identification of metabolic fluxes. Enzyme importance with respect to impact on ethanol productivity was ranked (Figure 2). It was found that an increase in ATP maintenance requirement decreased the ethanol productivity; this suggested that maintenance demand lowers ATP concentration, leading to an increased competition for ATP as well as limiting hexokinase activity and flux through glycolysis. To test this hypothesis, the analysis was repeated under the assumption that ATP saturates hexokinase. This hypothesis was simulated, assuming that most of the enzyme states of hexokinase were in complex form with the substrate, and that the elasticity of the enzyme with respect to ATP was near zero. Under this assumption, it was found that changes in ATP maintenance had no effect on glycolytic flux and ethanol productivity, confirming the hypothesis [42]. This example illustrates how methods such as ORACLE can also be used for hypothesis testing, without the need for a detailed kinetic model of the pathways.

\section{Concluding remarks}

Engineering complex metabolic pathways for the efficient production of biofuels and biochemicals is a challenging task, and the partial knowledge and uncertainty associated with the components of these pathways makes it difficult to advance the field. Although it will be hard to find some prophets and deities to help us with these complex problems, hopefully ORACLE and similar risk analysis methods will provide some much-needed 'prophetic' guidance.

The development of industrial microorganisms requires people from many disciplines working together: process engineers, chemical and biochemical engineers, microbial physiologists, biochemists, molecular biologists and geneticists. In such settings - when researchers are faced with complex problems - they usually start by focusing on what they know best. The use of uncertainty-based mathematical modeling can permit identification of metabolic engineering targets, without bias from previous experience, while simultaneously taking into account constraints from the process engineering and downstream design specifications.

Although MCA is a local sensitivity framework, it can serve as a starting point for the development of global sensitivity analysis methods. Such methods have been used extensively in other engineering fields, and they can be easily extended for application in biological systems [43]. Within the field of uncertainty and sensitivity analysis, methods exist for the analysis of uncertainty propagation. These methods can be used in the framework discussed above to identify which are the flux measurements or the 
enzymes whose uncertainty contributes the most toward uncertainty of the control coefficients. Identifying these sources of uncertainty can guide the investment of resources in the study of specific components of the pathways, such that uncertainty is reduced and the knowledge of the system-of-interest is vastly improved.

The nature of the data of uncertainty analysis also suggests the need for developments in various areas of computational biology, such as large-scale computation, statistical analysis and visualization toward productive communication among experts from diverse backgrounds. These areas have not been explored in the past, primarily because researchers in metabolic engineering and synthetic and systems biology have been overly concerned with accurate prediction driven by conventional engineering paradigms and practices. Hence, the demand for engineering microorganisms for fuels and chemicals will drive new developments and practices in mathematical and computational methods for metabolic engineering and synthetic biology.

\section{References}

1 Cameron, D.C. and Tong, I.T. (1993) Cellular and metabolic engineering - an overview. Appl. Biochem. Biotechnol. 38, 105-140

2 Fortman, J.L. et al. (2008) Biofuel alternatives to ethanol: pumping the microbial well. Trends Biotechnol. 26, 375-381

3 Alper, H. and Stephanopoulos, G. (2009) Engineering for biofuels: exploiting innate microbial capacity or importing biosynthetic potential? Nat. Rev. Microbiol. 7, 715-723

4 Tyo, K.E. et al. (2007) Expanding the metabolic engineering toolbox: more options to engineer cells. Trends Biotechnol. 25, $132-137$

5 Grotkjaer, T. et al. (2005) Comparative metabolic network analysis of two xylose fermenting recombinant Saccharomyces cerevisiae strains. Metab. Eng. 7, 437-444

6 Lee, S.K. et al. (2008) Metabolic engineering of microorganisms for biofuels production: from bugs to synthetic biology to fuels. Curr. Opin. Biotechnol. 19, 556-563

7 Vemuri, G.N. et al. (2007) Increasing NADH oxidation reduces overflow metabolism in Saccharomyces cerevisiae. Proc. Natl. Acad. Sci. U. S. A. 104, 2402-2407

8 Zamboni, N. and Sauer, U. (2009) Novel biological insights through metabolomics and C-13-flux analysis. Curr. Opin. Microbiol. 12, 553-558

9 Wiechert, W. (2001) C-13 metabolic flux analysis. Metab. Eng. 3, 195206

10 Gombert, A.K. et al. (2001) Network identification and flux quantification in the central metabolism of Saccharomyces cerevisiae under different conditions of glucose repression. J. Bacteriol. 183, $1441-1451$

11 Garcia, D.E. et al. (2008) Separation and mass spectrometry in microbial metabolomics. Curr. Opin. Microbiol. 11, 233-239

12 Rabinowitz, J.D. (2007) Cellular metabolomics of Escherchia coli. Expert Rev. Proteom. 4, 187-198

13 Bennett, B.D. et al. (2009) Absolute metabolite concentrations and implied enzyme active site occupancy in Escherichia coli. Nat. Chem. Biol. 5, 593-599

14 Boer, V.M. et al. (2010) Growth-limiting intracellular metabolites in yeast growing under diverse nutrient limitations. Mol. Biol. Cell 21, 198-211

15 Bailey, J.E. (1998) Mathematical modeling and analysis in biochemical engineering: Past accomplishments and future opportunities. Biotechnol. Progr. 14, 8-20

16 Fong, S.S. et al. (2005) In silico design and adaptive evolution of Escherichia coli for production of lactic acid. Biotechnol. Bioeng. 91, 643-648

17 Park, J.H. et al. (2008) Application of systems biology for bioprocess development. Trends Biotechnol. 26, 404-412
18 Park, J.M. et al. (2009) Constraints-based genome-scale metabolic simulation for systems metabolic engineering. Biotechnol. Adv. 27 , 979-988

19 Otero, J.M. and Nielsen, J. (2010) Industrial Systems Biology Biotechnol. Bioeng. 105, 439-460

20 Patil, K.R. et al. (2004) Use of genome-scale microbial models for metabolic engineering. Curr. Opin. Biotechnol. 15, 64-69

21 Burgard, A.P. et al. (2003) OptKnock: A bilevel programming framework for identifying gene knockout strategies for microbial strain optimization. Biotechnol. Bioeng. 84, 647-657

22 Pharkya, P. et al. (2004) OptStrain: a computational framework for redesign of microbial production systems. Genome Res. 14, 2367-2376

23 Klipp, E. (2007) Modelling dynamic processes in yeast. Yeast 24, $943-$ 959

24 Cascante, M. et al. (2002) Metabolic control analysis in drug discovery and disease. Nat. Biotechnol. 20, 243-249

25 Fell, D.A. (1992) Metabolic control analysis - a survey of its theoretical and experimental development. Biochem. J. 286, 313-330

26 Hatzimanikatis, V. and Bailey, J.E. (1996) MCA has more to say. J. Theor. Biol. 182, 233-242

27 Hatzimanikatis, V. and Bailey, J.E. (1997) Effects of spatiotemporal variations on metabolic control: Approximate analysis using (log)linear kinetic models. Biotechnol. Bioeng. 54, 91-104

28 Hatzimanikatis, V. et al. (1998) Application of mathematical tools for metabolic design of microbial ethanol production. Biotechnol. Bioeng. $58,154-161$

29 Moreno-Sanchez, R. et al. (2008) Metabolic control analysis: a tool for designing strategies to manipulate metabolic pathways. J. Biomed. Biotechnol. 2008, 597913

30 Heinrich, R. and Schuster, S. (1996) The Regulation of Cellular Systems, Springer

31 Hatzimanikatis, V. (1999) Nonlinear metabolic control analysis Metab. Eng. 1, 75-87

32 DeJongh, M. et al. (2007) Toward the automated generation of genomescale metabolic networks in the SEED. BMC Bioinformatics 8, 139

33 Henry, C.S. et al. (2009) iBsu1103: a new genome-scale metabolic model of Bacillus subtilis based on SEED annotations. Genome Biol. 10, R69

34 Feist, A.M. et al. (2009) Reconstruction of biochemical networks in microorganisms. Nat. Rev. Microbiol. 7, 129-143

35 Kumar, V.S. and Maranas, C.D. (2009) Growmatch: an automated method for reconciling in silico/in vivo growth predictions. PLoS Comput. Biol. 5, e1000308

36 Savageau, M.A. (1995) Michaelis-Menten mechanism reconsidered implications of fractal kinetics. J. Theor. Biol. 176, 115-124

37 Schnell, S. and Turner, T.E. (2004) Reaction kinetics in intracellular environments with macromolecular crowding: simulations and rate laws. Prog. Biophys. Mol. Biol. 85, 235-260

38 Jankowski, M.D. et al. (2008) Group contribution method for thermodynamic analysis of complex metabolic networks. Biophys. J. 95, 1487-1499

39 Ayyub, B.M. and Klir, G.J. (2006) Uncertainty Modeling and Analysis in Engineering and the Sciences, Chapman and Hall/CRC

40 Wang, L.Q. et al. (2004) Metabolic control analysis under uncertainty: framework development and case studies. Biophys. J. 87, 3750-3763

41 Wang, L.Q. and Hatzimanikatis, V. (2006) Metabolic engineering under uncertainty. I: framework development3. Metab. Eng. 8, 133-141

42 Wang, L.Q. and Hatzimanikatis, V. (2006) Metabolic engineering under uncertainty - II: analysis of yeast metabolism. Metab. Eng. 8, 142-159

43 Kiparissides, A. et al. (2009) Global sensitivity analysis challenges in biological systems modeling. Ind. Eng. Chem. Res. 48, 7168-7180

44 Calvetti, D. et al. (2008) Dynamic Bayesian sensitivity analysis of a myocardial metabolic model. Math. Biosci. 212, 1-21

45 Heino, J. et al. (2010) METABOLICA: a statistical research tool for analyzing metabolic networks. Comput. Methods Programs Biomed. $97,151-167$

46 Occhipinti, R. et al. (2009) Astrocytes as the glucose shunt for glutamatergic neurons at high activity: an in silico study. $J$. Neurophysiol. 101, 2528-2538

47 Gutenkunst, R.N. et al. (2007) Universally sloppy parameter sensitivities in systems biology models. PLoS Comput. Biol. 3, $1871-1878$ 


\section{Opinion}

Trends in Biotechnology Vol.xxx No.x

48 Alves, R. and Savageau, M.A. (2000) Systemic properties of ensembles of metabolic networks: application of graphical and statistical methods to simple unbranched pathways. Bioinformatics 16, 534-547

49 Pritchard, L. and Kell, D.B. (2002) Schemes of flux control in a model of Saccharomyces cerevisiae glycolysis. Eur. J. Biochem. 269, 3894-3904

50 Rizk, M.L. and Liao, J.C. (2009) Ensemble modeling for aromatic production in Escherichia coli. PLoS One 4, e6903

51 Tran, L.M. et al. (2008) Ensemble modeling of metabolic networks. Biophys. J. 95, 5606-5617

52 Contador, C.A. et al. (2009) Ensemble modeling for strain development of L-lysine-producing Escherichia coli. Metab. Eng. 11, 221-233

53 Liebermeister, W. and Klipp, E. (2006) Bringing metabolic networks to life: convenience rate law and thermodynamic constraints. Theor. Biol. Med. Model. 3, 41

54 Liebermeister, W. and Klipp, E. (2006) Bringing metabolic networks to life: integration of kinetic, metabolic, and proteomic data. Theor. Biol. Med. Model. 3, 42
55 Goldberg, R.N. et al. (2004) Thermodynamics of enzyme-catalyzed reactions - a database for quantitative biochemistry. Bioinformatics 20, 2874-2877

56 Goldberg, R.N. et al. (2007) Thermodynamics of enzyme-catalyzed reactions: Part 7 - 2007 update. J. Phys. Chem. Ref. Data 36, 1347-1397

57 Beard, D.A. and Qian, H. (2008) Chemical Biophysics: Quantitative Analysis of Cellular Systems, Cambridge University Press

58 Henry, C.S. et al. (2007) Thermodynamics-based metabolic flux analysis. Biophys. J. 92, 1792-1805

59 Henry, C.S. et al. (2006) Genome-scale thermodynamic analysis of Escherichia coli metabolism. Biophys. J. 90, 1453-1461

60 Chen, J.Y. and Lonardi, S. (2009) Biological Data Mining, Chapman and Hall/CRC

61 Conover, W.J. (1998) Practical Nonparametric Statistics, Wiley

62 Silverman, B.W. (1986) Density Estimation for Statistics and Data Analysis, Chapman and Hall/CRC 